JOURNAL OF SYNCHROTRON RADIATION

ISSN 1600-5775

Received 22 June 2015

Accepted 22 June 2015

Keywords: ANKA; KIT; user facility.

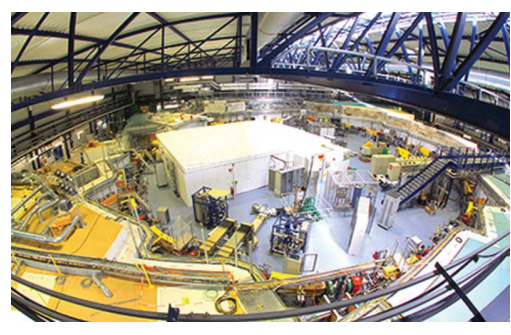

(C) 2015 International Union of Crystallography

\section{On the ANKA decision: a response to Braun's Just for us?}

\author{
Volker Saile* \\ Division of Physics and Mathematics, Karlsruhe Institute of Technology, Kaiserstrasse 12, Karlsruhe 76131, Germany. \\ *Correspondence e-mail: volker.saile@kit.edu
}

KIT's Executive Board has decided to discontinue the operation of the synchrotron radiation facility ANKA within category LK-II (user facility) of the Helmholtz Association and continue operation in LK-I (research). The KIT Senate confirmed this decision. Some aspects of the background and the rationale leading to this change are explained.

In March this year, KIT's Executive Board decided to discontinue the general user operation of ANKA as defined by the so-called LK-II (Leistungskategorie II) rules of the Helmholtz Association and to continue operation in LK-I, the general research category. This decision was confirmed by the KIT Senate in May 2015.

Understandably, this decision has raised concerns and confusion in the synchrotron radiation community and beyond. Dr Braun's Letter to the Editor (Braun, 2015) is a vivid testimony of these valid concerns. In my response I refrain from answering Dr Braun's questions and statements one by one; also, I will not justify or criticize decisions of KIT's Executive Board or the KIT Senate but rather explain the background and reasons for this situation.

The two dominant aspects in the ANKA discussion are quite simple: money and strategy. As for the first one, ANKA has always been underfunded over the past 15 years due to a systematic problem in the funding scheme, you may also call it a birth defect, and this situation could not be improved over time; just the contrary, due to the complex funding mechanisms of the German federal system, a significant part of the budget was lost. In spite of this deficiency in base funding a highly motivated ANKA team was able to enlarge the buildings, add beamlines and laboratories, last but not least by attracting additional financial support through grants, contracts and other sources. But the precarious situation with the base funding stays the same also for the current Helmholtz period 2015 to 2019. On the other side, for a LK-II user operation ANKA has to guarantee significantly more than $50 \%$ of the available beam time for external (external to the Helmholtz Association) users, a 24/7 operation of a large number of beamlines, and sufficient support staff following national and international standards. The ANKA directors declared that they cannot fulfill these commitments of a LK-II facility within the current budget constraints. This led the KIT Executive Board to the decision to discontinue operation under LK-II and to continue as a research facility under LK-I; the KIT Senate confirmed this decision. Just to make this point very clear: in all the numerous meetings and discussion I attended, Dr Braun's 'just for us' access policy was never considered as a desirable option for ANKA or KIT, just the contrary.

The second aspect, strategy, addresses the longer-term plans for ANKA including the life span of the storage ring. As also evident from Dr Braun's references of the early years, ANKA was originally built for industrial applications and only later converted into a user facility for the research community. The implications of the original concept, political ones and technical ones, haunted ANKA over the past 15 or more years. The latter include a low-energy injector, a too high emittance in comparison with state-of-theart synchrotron radiation sources, and a very limited number of straight sections for undulators. In spite of strong recommendations of ANKA's advisory boards, MAC and SAC, the Forschungszentrum Karlsruhe and later the KIT were unable to attract or generate resources for a major upgrade of the accelerator system and the prospects for finding such support are lower today than ever. Nevertheless, even though not being counted in the top league of third- or fourth-generation sources and lacking resources, 
ANKA with its highly motivated team has supported the synchrotron radiation community in an exemplary way over the past years. But it is also true that ANKA's competitiveness is limited and will deteriorate further in future with the upgrades of already today more powerful rings and with new facilities coming online in Europe and elsewhere.

ANKA is a success story. The in-house and external users have, in combination with the excellent team that is responsible for building and running the facility, contributed significantly to the progress in synchrotron radiation research including technology developments. Dr Braun mentions the recent breakthrough in superconducting undulator technology and one could add many more examples for internationally recognized results in accelerator physics and technology, beamline technology and in applications in materials science, life sciences and also in providing solutions for industry.

What next? KIT must develop a new concept for ANKA for the funding period 2015 to 2019 and beyond. It must also take into account specific requirements such as the operation of beamlines with unique capabilities not available at or not transferable to other sources, such as the ones for actinide research, catalysis and X-ray lithography, to name a few. Drafts for such a concept are being circulated within KIT but more time is needed for discussions in KIT but also for involving our advisory boards and external experts.

I personally welcome all input from the user community at large via e-mail or phone or personal visits.

\section{Acknowledgements}

The author has been working in synchrotron radiation since the early seventies of the last century. Currently, he serves as the Head of KIT's Division 5 - Physics and Mathematics. Since ANKA is one of the units in this Division he assumes responsibility for providing additional input on the ANKA discussion in response to Dr Braun's Letter to the Editor.

\section{References}

Braun, A. (2015). J. Synchrotron Rad. 22, 1327-1328. 